\title{
Aligning Researcher Practice to Support Public Access to Data
}

\section{Surya K. Mallapragada, Associate Vice President for Research, Iowa State University}

7 here is a national move towards open science and open enquiry. The Royal Society summary report (2012), places "open enquiry at the heart of the scientific enterprise". The report outlines the different advantages that open access brings, including enabling change, facilitating new ways of doing science, allowing new collaborations to flourish, enabling better communication of science with the public, enhancing reproducibility and quality and impacting transparency and accountability.

Studies have shown that open science can facilitate research and can help researchers succeed by improving citation rates. The extent of benefit was found to vary across disciplines, as seen in Figure 1 (from McKiernan et al., 2016).

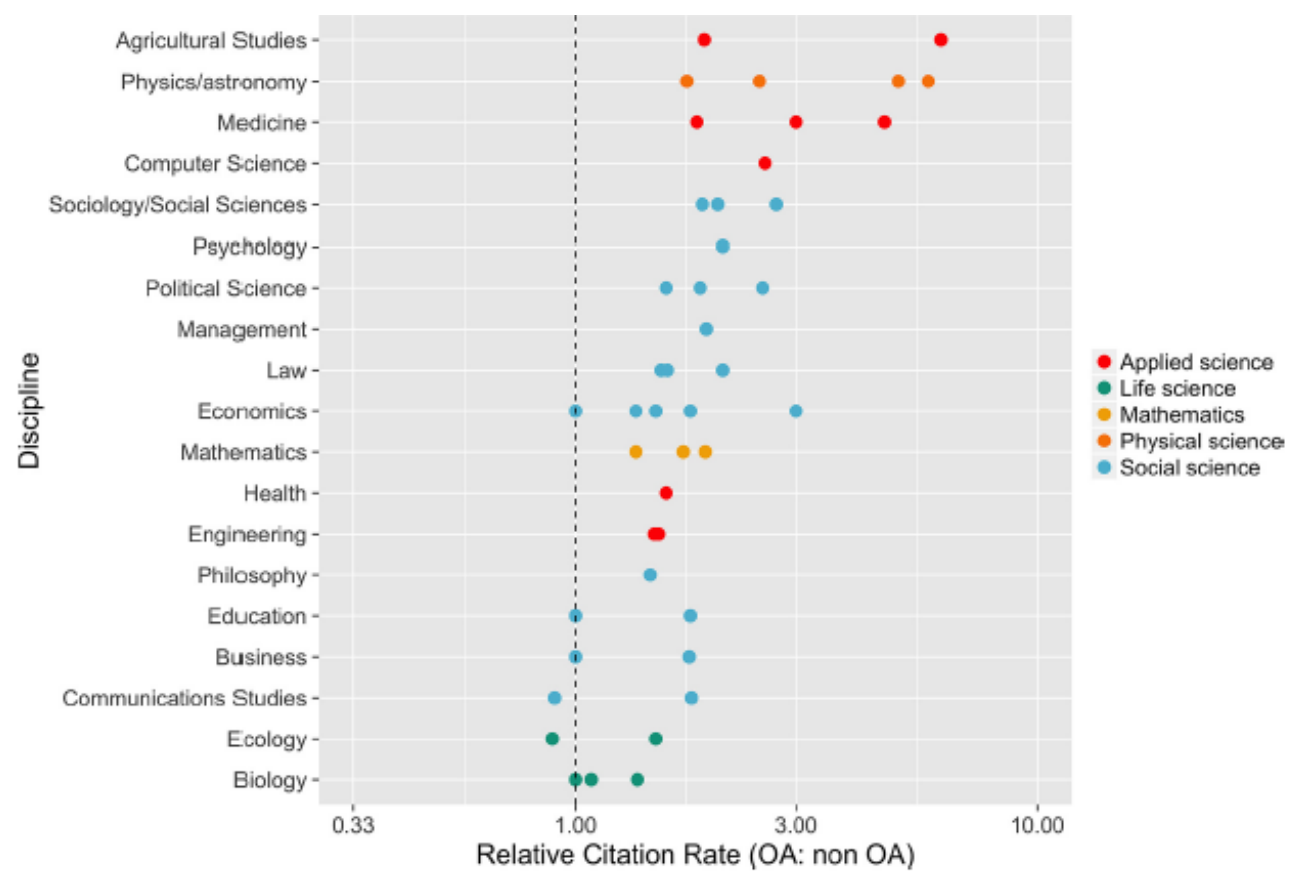

Figure 1. Open access articles get more citations. The relative citation rate is defined as the mean citation rate of open access articles divided by the mean citation rate of non-open access articles. (Reproduced from McKiernan et al, eLIFE, 2016; 5:e16800. License: https://creativecommons.org/licenses/by/4.0/legalcode). 
There has been a recent increase in open access policies in the US (Figure 2), and the Holdren memo (2013) mandates public access to research products developed with federal funding.

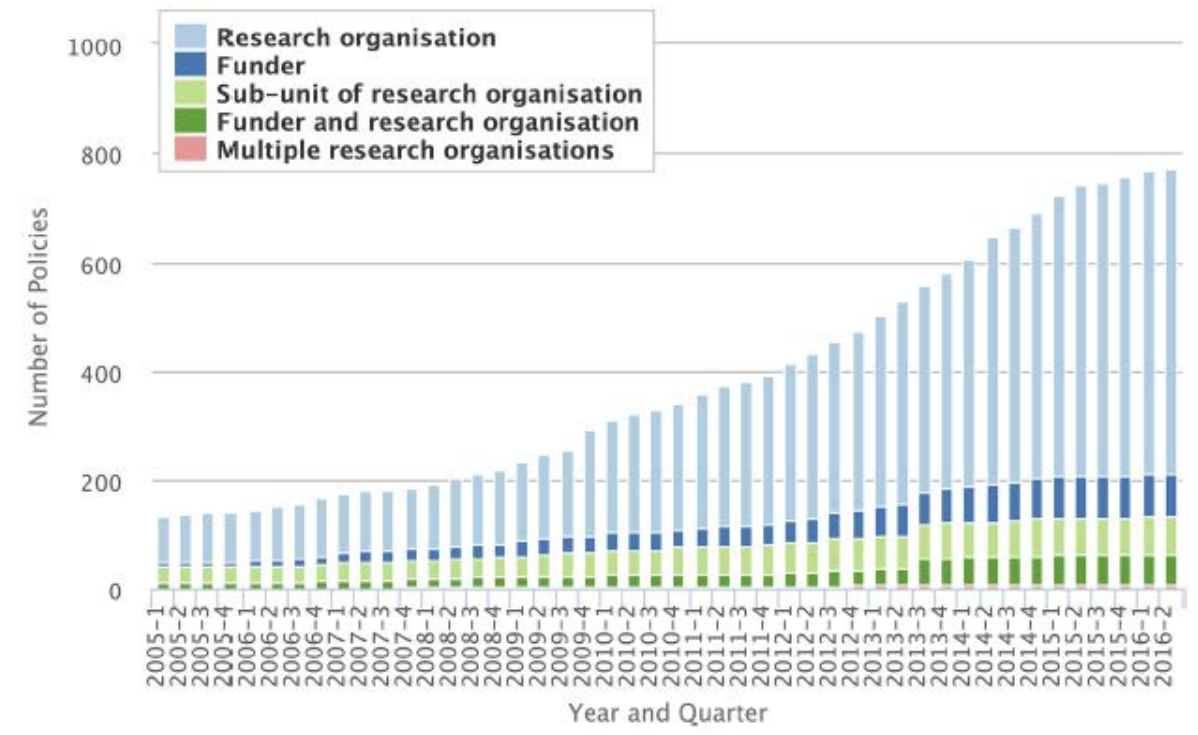

Figure 2. Recent increase in open access policies (Reproduced from McKiernan et al., eLIFE, 2016; 5:e16800. License: https://creativecommons.org/licenses/by/4.0/legalcode)

While the resources and systems for openly sharing publications are well developed, the policies associated with data sharing are less well defined, and sometimes contradictory. Open access to data will be effective only if there are common standards for communicating data and there is a cohesive and uniform strategy used, so that data is usable by others and sharing data is a criterion for career progression. Mayernik (2017) has conducted an interesting analysis of the likelihood of research practices related to open data policies initiatives achieving the goals of open data policy initiatives. Figure 3 presents a model of open data distinguishing between hard and soft accountability as well as different levels of transparency, to arrive at the relative likelihood in each case that research practices will achieve the goals of broad accessibility and usability of data. 


\section{TRANSPARENCY}

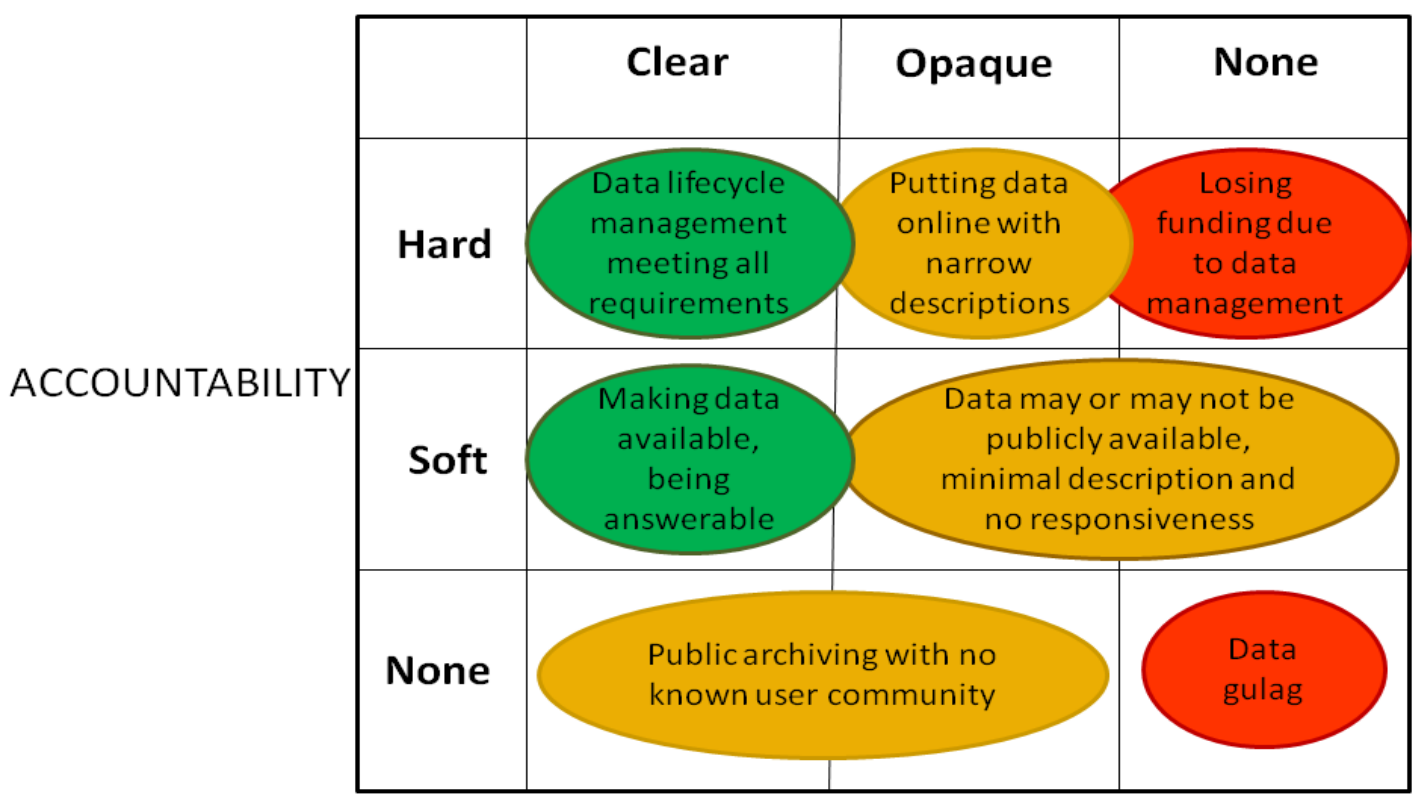

Figure 3. Color scheme shows likelihood (green to red) of research practices related to open data policy initiatives achieving the goals of broad accessibility and usability of data (Reproduced with permission from Mayernik, 2017).

The Open Science Framework is an example of a workflow management system designed to help researchers in this regard (http://osf.io). Data sharing is the focus of an AAU-APLU Public Access Working Group co-chaired by Dr. Sarah Nusser, Vice President for Research at Iowa State University, that is working on common goals for data sharing, federal agency recommendations and guidance for research institutions. At Iowa State University, the implementation is being coordinated across the Library, IT services and the Office of the Vice President for Research, aided by a faculty committee to provide faculty perspective on building a rigorous process for data sharing. Some key questions to consider as we develop institutional policies and practices are to see what the purpose of sharing is, what data should be shared, what the standard should be for documenting data, different options for storing the data, and finally how we can train researchers to adopt this new mindset.

\section{References}

1. "Science as an open enterprise", The Royal Society, London. 2012

2. "How open science helps researchers succeed", McKiernan, E.C., Bourne, P. E., Brown, C.T. et al., eLIFE, 2016; 5:e16800

3. "Open data: Accountability and transparency", Mayernik, M.S., Big Data and Society, 1-5 (2017) 